\title{
Presentation of a Rare Case of Bilateral Lumbar Synovial Cysts
}

\author{
Konstantinos Violaris", Maria Karakyriou \\ Nicosia General Hospital, Nicosia, Cyprus \\ Email: *kaviola@hotmail.com
}

Received December 8, 2011; revised December 22, 2011; accepted January 4, 2012

\begin{abstract}
Purpose: To report a quite rare case of bilateral symptomatic synovial cysts of the lumbar spine. Surgical resection is usually the cure for this condition. Methods: A 65 year old female patient came to our department, complaining for severe lumbar and sciatic pain. MRI and CT were used to diagnose bilateral synovial cysts. Because of the intensity of symptoms, she was subjected to surgery. Laminectomy and cyst resection were performed. Results: The patient showed complete resolution of pain after the resection of the cysts. Conclusion: Although rare, bilateral synovial cysts may be the cause of severe lumbar pain. In cases of failure of conservative treatment, surgical resection offers good results.
\end{abstract}

Keywords: Bilateral Synovial Cysts; Surgical Resection

\section{Introduction}

The formation of a unilateral synovial cyst is a seldom cause of back pain and sciatica in the elderly [1]. Bilateral symptomatic synovial cysts of the lumbar spine are very rare [1-4]. We report a case of a 65 year old female patient, with bilateral symptomatic synovial cysts at L4 L5 level. She presented with severe lumbar pain and bilateral sciatica. Because of the intensity of pain she had severe limitation of motion and walking. She underwent surgical resection of the cysts and presented complete resolution of pain.

\section{Methods}

This 65 year old obese female patient presented bilateral sciatica for four months. She complained for pain that started on the right side and gradually moved to the left side, too. She was administrated with analgesics and physiotherapy but found no pain relief. Her walking worsened and she was limited in her house. She did not response even to steroids. As she gradually deteriorated, the pain persisted throughout the night. Physical examination revealed normal muscle strength of lower extremities. The lumbar spine showed full flexion but mildly restricted extension. There was no scoliosis, listing or shifting of the spine on forward flexion. The straight legraising test was positive on both sides. Sensory testing revealed hypoesthesia of bilateral L5 dermatomes. The Achilles reflexes were markedly decreased bilaterally.

Magnetic resonance imaging (Figures 1, 2) revealed

"Corresponding author. well circumscribed bilateral lesions in the dorsolateral portion of the spinal canal at L4 - L5 (more prominent on the right side). The lesions appeared hypointense on axial T1-weighted images. T2-weighted images demonstrated a hyperintense central region and a hypointense rim. Contrast CT showed the lesions, enhancing with contrast.

As symptoms would not resolve, she was offered the choice of surgery. Laminectomy of L4 was performed. Excessive degeneration of the spine was obvious. Whitish connective tissue, extending to the adjacent ligamentum flavum was found. A lesion was identified dorsal to the shoulder of the right L5 nerve root, originating at the facet joint. The lesion was cystic, but not entirely as we expected; it was mainly consisted of strong connective tissue. This created severe stenosis of the spinal canal, as it occupied the space to the midline. On the left side, a similar formation was found at a lesser extend. There was no disc herniation at L4 - L5. The involved solid and cystic tissues were resected with a partial facetectomy. This was achieved with extreme caution, as strong adhesions of the dura to the synovial were present.

After surgery, the patient recovered well. Her lowback and leg pain was relieved, and she was able to walk the next morning. She mentioned that the numbness of legs diminished. Within a week after surgery she went back to normal activities. During 1 year of follow-up, she had no recurrent symptoms.

Pathological examination of the surgical specimen revealed findings consistent with synovial cyst. The pathologists noticed a structure that contained pieces of connective tissue and dense fibrin. The cyst wall was com- 


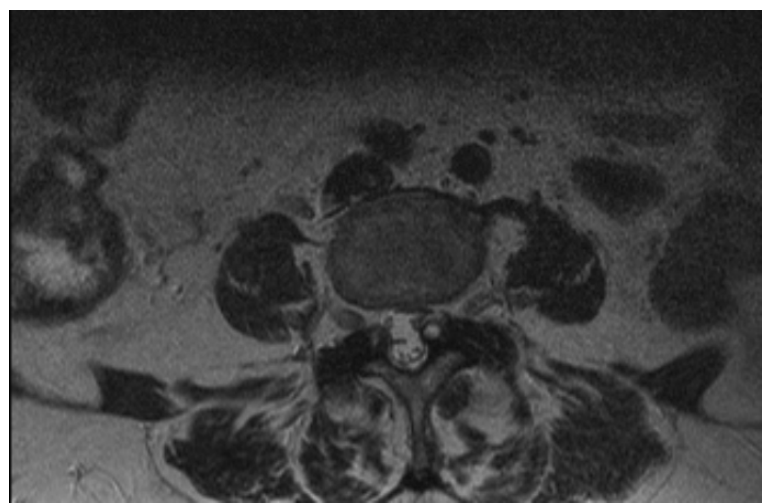

Figure 1. T2 weighted MRI, transverse section. Bilateral synovial cysts, more prominent on the left side compromise nerve roots.

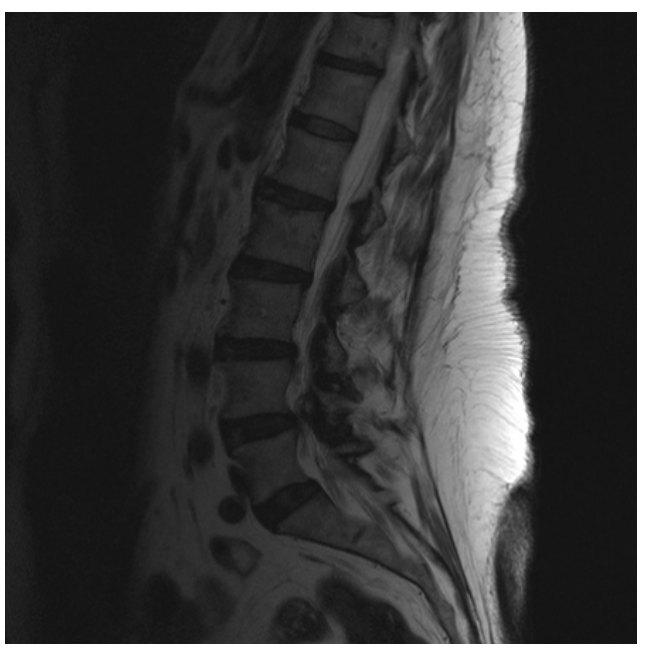

Figure 2. T2 weighted MRI, sagital view. A synovial cyst appears at L4/L5 level, creating canal stenosis laterally.

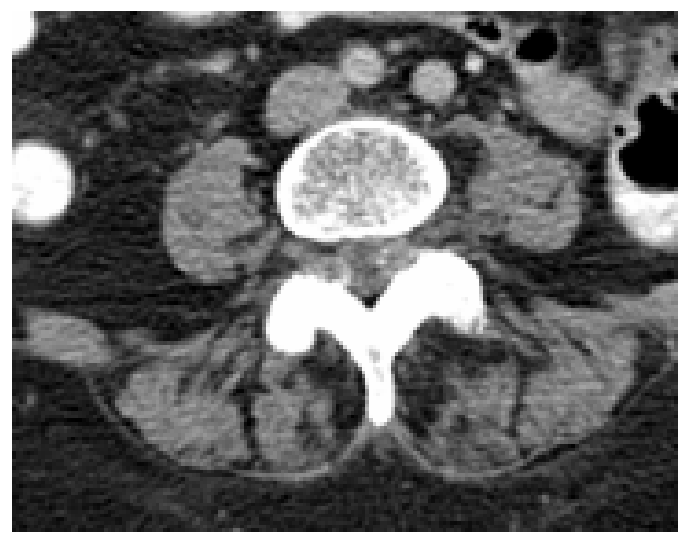

Figure 3. Contrast CT. Bilateral synovial cysts enhancing, more prominent on the left side are clearly seen.

posed of connective tissue and fibrous cartilage.

\section{Discussion}

A synovial cyst is a fluid-filled sac that develops as a re- sult of degeneration of the facet joint in the spine [1,5]. Synovial cysts have alternatively been referred to as ganglion cysts or juxta-articular cysts, depending on the presence of a true synovial lining [6,7]. They represent a rare cause of lumbar pain and sciatica. The clinical pictures in patients with synovial cysts of the lumbar spine vary considerably. The symptoms differ according to the size and location of the cyst in relation to neural structures $[3,5]$. Synovial cysts are located at a number of different sites such as the dorsal midline with involvement of the dura mater, the inner aspect of the ligamentum flavum without attachment to the facet, the spinal canal with attachment to the facet through the interlaminar space, or the ligamentum flavum itself. They appear more commonly in patients older than 65 years old and are not often seen in patients younger than 45 [1]. It is typically a process that usually happens in the lumbar spine, and most commonly develops at the L4 - L5 level (rarely at L3 - L4). The aetiology of intraspinal synovial cyst is unknown, but may relate to either herniation of synovium from the facet joint, or to mucinous degeneration of the connective tissue adjacent to the joint. Histological findings of myxoid degeneration, microcystic change, calcification and hemosiderin deposits suggest that chronic microtrauma with occasional focal hemorrhage may play a major role in their creation [8]. The fluid-filled sac creates pressure inside the spinal canal and this in turn can give a patient all the symptoms of stenosis of the spine [9]. The pain probably comes from the venous blood around the nerves not being able to drain and this leads to pain and irritation of the nerves.

Spinal synovial cysts are usually diagnosed with CT and MRI $[10,11]$. Typically, these cysts appear on CT as posterolateral extradural masses that may be partially calcified or contain gas. On MRI, these cysts appear as well circumscribed structures, often without evidence of association with a facet joint. The signal intensity varies greatly depending on the characteristics of the cyst. In general, on Tl- and T2-weighted images the cystic cavity is hyperintense compared to cerebrospinal fluid because the material within usually contains some protein. In contrast, cysts with wall calcification may produce low-intensity signals. In most cases, administration of gadolinium contrast results in uniform rim enhancement due to the presence of chronic inflammation.

Our patient presented with low-back and bilateral leg pain that limited her daily activities. The problem was not responsive to analgesics and anti-inflammatory drugs. The straight leg-raising test was positive, and she exhibited hypoesthesia in the related dermatomes. Radiological investigation showed bilateral synovial cysts compressing both L5 nerve roots. After the failure of conservative treatment, surgery was the treatment of choice in this case. The patient's pain was completely relieved by 
the operation.

\section{Conclusion}

Intraspinal synovial cysts are seldom lesions that most often arise in the lumbar region. Bilateral sciatica is rarely caused by bilateral synovial cysts. These cysts should always be included in the differential diagnosis for lumbar spinal disease. Symptoms such as sciatica and lumbar pain usually mimic those of intervertebral disc herniation. Surgical decompression and excision has excellent results. Even in very rare cases, such as our case report, establishing the definitive diagnosis preoperatively is important in planning the most appropriate treatment.

\section{REFERENCES}

[1] B. A. Freedman, T. L. Bui and S. T. Yoon, "Diagnostic Challenge: Bilateral Infected Lumbar Facet Cysts-A Rare Cause of Acute Lumbar Spinal Stenosis and Back Pain,” Journal of Orthopaedic Surgery and Research, Vol. 5, No. 1, 2010, p. 14. doi:10.1186/1749-799X-5-14

[2] A. Gadgil, S. Eisenstein, A. Darby and V. C. Pullicino, "Bilateral Symptomatic Synovial Cysts of the Lumbar Spine Caused by Calcium Pyrophosphate Deposition Disease: A Case Report,” Spine, Vol. 27, No. 19, 2002, pp. 428-431.

[3] A. Bozzao, R. Floris, C. Fraioli, L. Ticca and P. Simonetti, "Relapsing-Remitting Bilateral Synovial Cysts of the Lumbar Spine. A Case Report,” Diagnostic Neuroradiology, Vol. 43, No. 12, 2001, pp. 1076-1078. doi:10.1007/s002340100642

[4] B. Coulier, J. Ghosez and P. Mailleux, "Computed Tomography Diagnosis of Clinically Well-Tolerated Bilateral Lumbar Vertebral Synovial Cysts, Journal Belge de
Radiologie-Belgisch Tijdschrift voor Radiologi, Vol. 81, No. 3, 1998, pp. 141-143.

[5] N. E. Epstein, "Lumbar Synovial Cysts: A Review of Diagnosis, Surgical Management, and Outcome Assessment," Journal of Spinal Disorders \& Techniques, Vol. 17, No. 4, 2004, pp. 321-325. doi:10.1097/01.bsd.0000096267.75190.eb

[6] J. de Beukelaar, A. Dallenga, G. de Jong, K. de Winker and S. L. Bakker, "Juxta Facet Cyst of the Lumbar Spine: An Uncommon Cause of Lumbar Radiculopathy,” Nederlands Tijdschrift Voor Geneeskunde, Vol. 154, 2010, p. A1673.

[7] R. Deinsberger, E. Kinn and K. Ungersböck, "Microsurgical Treatment of Juxta Facet Cysts of the Lumbar Spine," Journal of Spinal Disorders \& Techniques, Vol. 19, No. 3, 2006, pp. 155-160. doi:10.1097/01.bsd.0000188660.31212.83

[8] K. Gong, Z. Wang, M. Chen and Z. Luo, "Diagnosis and Surgical Management of Intraspinal Hemorrhagic Juxtafacet Cysts in Lumbar Spine: Experience of Eight Cases,” Neurology India, Vol. 58, No. 2, 2010, pp. 288-291. doi:10.4103/0028-3886.63788

[9] R. Acharya, R. Patwardhan, D. Smith, B. Willis, M. Fowler and A. Nand, "Intraspinal Synovial Cysts: A Retrospective Study,” Neurology India, Vol. 54, No. 1, 2006, pp. 38-41.

[10] W. Yuh, J. Drew, J. Weinstein, C. McGuire, T. Moore, M. Kathol and G. El-Khoury, "Intraspinal Synovial Cysts. Magnetic Resonance Evaluation,” Spine, Vol. 16, No. 7, pp. 740-745. doi:10.1097/00007632-199107000-00009

[11] N. J. Bureau, P. A. Kaplan and R. G. Dussault, "Lumbar Facet Joint Synovial Cyst: Percutaneous Treatment with Steroid Injections and Distention-Clinical and Imaging Follow-Up in 12 Patients,” Radiology, Vol. 221, No. 1, 2001, pp. 179-185. doi:10.1148/radiol.2211010213 